\title{
Clinical Decision Making Regarding Benzodiazepine Use in PTSD Treatment
}

\author{
nancy bernardy ${ }^{1}$, Erin Barnett ${ }^{2}$, Brian Lund ${ }^{3}$, Bruce Alexander $^{3}$, Louise Parker ${ }^{4}$, Aaron \\ Jenkyn $^{1}$, and Matthew Friedman ${ }^{1}$ \\ ${ }^{1}$ White River Junction VA Medical Center \\ ${ }^{2}$ Dartmouth College Geisel School of Medicine \\ ${ }^{3}$ Iowa City VA Medical Center \\ ${ }^{4}$ University of Massachusetts Boston
}

December 1, 2020

\begin{abstract}
i. Rationale, Aims and Objectives: Despite guideline recommendations against their use, clinicians prescribe benzodiazepines for various symptoms to patients with posttraumatic stress disorder (PTSD). Clinicians' reasons in making these decisions are not fully understood. This qualitative study sought to characterize factors identified by prescribing clinicians in clinical decision making in PTSD regarding the use of benzodiazepines. ii. Methods: The descriptive study involved semi-structured interviews with 26 prescribing clinicians across thirteen VA medical centers. Our overall aim in the study was to explore clinicians' benzodiazepine practices in veterans with a PTSD diagnosis. We audio-recorded, transcribed, and analyzed the interviews using grounded theory methodology. iii. Results: Facilitators and barriers that contribute to benzodiazepine prescribing to veterans with PTSD included organizational, provider, and patient aspects. Most providers interviewed indicated that they inherited patients already on these medications initiated by other clinicians. These providers, as well as others interviewed, voiced concerns that tapering benzodiazepines may cause more harm than the risks of maintenance, particularly in older patients. Clinicians who noted consistent treatment practices among their hospital colleagues found it easier to decrease both new and maintenance benzodiazepine prescribing. iv. Conclusions: Patients with PTSD at increased risk of harms, such as older patients, are still receiving benzodiazepines suggesting that innovative solutions are now needed to decrease use. Specific protocols for inherited patient caseloads, increased dissemination of effective psychotherapies for symptoms such as insomnia and anxiety and the use of direct to consumer educational materials should help to foster needed culture change and increased evidence-based PTSD practice.
\end{abstract}

\section{Clinical Decision Making Regarding Benzodiazepine Use in PTSD Treatment}

Running Title: Clinical Decision Making

Nancy C. Bernardy $\mathrm{PhD}_{1}$, Erin Barnett $\mathrm{PhD}_{2}$, Brian C. Lund PharmD $\mathrm{D}_{3}$, Bruce Alexander

PharmD 4 , Louise E. Parker $\mathrm{PhD}_{5,6}$ Aaron Jenkyn $\mathrm{BA}_{7}$, Matthew J. Friedman MD, $\mathrm{PhD}_{8}$

1 Health Science Specialist, National Center for PTSD, Veterans Affairs Medical Center, White River Junction, VT and Associate Professor, Department of Psychiatry, The Geisel School of Medicine at Dartmouth, Hanover, NH

2 Assistant Professor of Psychiatry and The Dartmouth Institute for Health Policy and Clinical Practice, The Geisel School of Medicine at Dartmouth, Hanover, NH 
3 MS, Core Investigator, Center for Comprehensive Access \& Delivery Research and Evaluation, Iowa City Veterans Affairs Health Care System, Iowa City, IA

${ }_{4}$ Contract Data Manager, Center for Comprehensive Access \& Delivery Research and Evaluation, Iowa City Veterans Affairs Health Care System, Iowa City, IA

5 University of Massachusetts Boston, Department of Management, Boston, MA

6 VA Quality Enhancement Research Initiative (QUERI) Program for Team-Based Behavioral Health, Department of Veterans Affairs

${ }_{7}$ Research Coordinator, National Center for PTSD, Veterans Affairs Medical Center, White River Junction, VT

${ }_{8}$ Senior Advisor, National Center for PTSD, Veterans Affairs Medical Center, White River Junction, VT and Departments of Psychiatry, The Geisel School of Medicine at Dartmouth, Hanover, NH

Corresponding author and address for reprints: Dr. Nancy Bernardy, National Center for PTSD (116D), White River Junction VA Medical Center, 215 North Main, White River Junction, VT 05001, phone 802/296-5132, fax 802/296-5135, Nancy.Bernardy@va.gov

Abstract count 251

Word count 4325

3 Tables

\section{ABSTRACT}

1. Rationale, Aims and Objectives: Despite guideline recommendations against their use, clinicians prescribe benzodiazepines for various symptoms to patients with posttraumatic stress disorder (PTSD). Clinicians' reasons in making these decisions are not fully understood. This qualitative study sought to characterize factors identified by prescribing clinicians in clinical decision making in PTSD regarding the use of benzodiazepines.

2. Methods: The descriptive study involved semi-structured interviews with 26 prescribing clinicians across thirteen VA medical centers. Our overall aim in the study was to explore clinicians' benzodiazepine practices in veterans with a PTSD diagnosis. We audio-recorded, transcribed, and analyzed the interviews using grounded theory methodology.

3. Results: Facilitators and barriers that contribute to benzodiazepine prescribing to veterans with PTSD included organizational, provider, and patient aspects. Most providers interviewed indicated that they inherited patients already on these medications initiated by other clinicians. These providers, as well as others interviewed, voiced concerns that tapering benzodiazepines may cause more harm than the risks of maintenance, particularly in older patients. Clinicians who noted consistent treatment practices among their hospital colleagues found it easier to decrease both new and maintenance benzodiazepine prescribing.

4. Conclusions: Patients with PTSD at increased risk of harms, such as older patients, are still receiving benzodiazepines suggesting that innovative solutions are now needed to decrease use. Specific protocols for inherited patient caseloads, increased dissemination of effective psychotherapies for symptoms such as insomnia and anxiety and the use of direct to consumer educational materials should help to foster needed culture change and increased evidence-based PTSD practice.

\section{INTRODUCTION}

Over the last decade, the US Department of Veterans Affairs (VA) has implemented initiatives to promote effective pharmacotherapy treatment of posttraumatic stress disorder (PTSD). Prominent among these has been the national dissemination of academic detailing (AD), an educational outreach intervention led by clinical pharmacists that offers best practice in a clinical area such as PTSD by trained educators in the practitioner's office. $^{1,2} \mathrm{AD}$ enables pharmacists to use a developed pharmacotherapy dashboard to review 
a prescribing clinician's caseload and medications prescribed for specific patients. Another resource is the Psychotropic Drug Safety Initiative, the PDSI, a national psychopharmacology quality improvement program that offers clinicians support by providing hospital data on prescribing measures and didactic lectures on specific medications. ${ }^{3}$ Rather than the focus on individual patients seen in $\mathrm{AD}$, the PDSI focuses on specific pharmacotherapy hospital metrics. Both initiatives have concentrated on veterans with PTSD who are most at risk from benzodiazepine use including the older veteran population aged 65 and over. The two programs have been largely successful with only about $10 \%$ of veterans with PTSD still receiving benzodiazepine prescriptions ${ }^{4}$ a decrease from $30 \%$ we observed a decade earlier. ${ }^{5}$ The decrease is encouraging, yet the $10 \%$ of veterans with PTSD who are still prescribed drugs from this class of medications, half of whom are over the age of 65, suggests a knowledge gap between clinician decision making and recognition of the evidence of harms from chronic benzodiazepine use. It further supports the need to examine the reasons providers prescribe benzodiazepines to high risk patients to help educators develop new strategies to decrease use.

The 2017 joint VA/DoD (Department of Defense) Clinical Practice Guideline (CPG) for PTSD ${ }^{6}$ offers treatment recommendations to guide clinicians in clinical decision making. New in 2017 to the guideline is a recommendation of trauma-focused cognitive behavioral psychotherapies as first-line PTSD treatment. Four specific antidepressants are recommended as pharmacologic monotherapy and the revised CPG again recommends against the use of benzodiazepines due to a lack of efficacy and safety concerns.

Although there are concerns about both short-term and long-term benzodiazepine use, the documented harms of chronic benzodiazepine use (typically $>3$ months) are well-established and include accidents, falls, hip fractures and cognitive dysfunction. ${ }^{7}$ These risks are highest in older adults who comprise the majority of patients with PTSD treated in the VA. ${ }^{8}$ There is some evidence that chronic benzodiazepine use, especially in patients with PTSD because of its own unique risk, can lead to the development of dementia; an obvious concern among older patients. ${ }^{9,10}$ Recent research also notes immune system harmful effects, a risk that is particularly relevant now due to Covid-19. ${ }^{11}$

Concerns around decision making regarding benzodiazepine use in patients with PTSD, however, are not unique to the VA. The prescribing of benzodiazepines nationally in ambulatory care has increased substantially with primary care visits in the U.S. accounting for about half of all benzodiazepine visits. ${ }^{12}$ Previous work examined reasons for prescribing benzodiazepines by primary care providers working in the community and found these clinicians doubted the risks of chronic benzodiazepine use and were pessimistic that patients would agree to tapers. ${ }^{13}$ Recent research also noted increased rates of benzodiazepine prescribing in community mental health settings to patients with co-occurring mental health and substance use disorders, a practice that should be avoided. ${ }^{14}$ Although our work is concerned with better understanding decision making regarding benzodiazepine prescribing among US VA clinicians, we believe that it provides general lessons for clinical decision making for non-veteran patients with PTSD in the US and also in other countries, particularly those with national healthcare and insurance systems. Similar to such national systems, VA which is comprised of a nation-wide system of hospitals and outpatient facilities with a shared mission, caring for the nations' veterans; and as such, is able to implement system-wide clinical policies and mandates for improving care and rendering it more compliant with evidence.

\section{METHODS}

\subsection{Design}

This qualitative study is part of an ongoing comprehensive project examining the process of improving the delivery of evidence-based PTSD care. This early work using grounded theory methodology ${ }^{15}$ explored prescribing clinicians' perceptions of adherence to the CPG recommendation regarding prescribing of benzodiazepines to patients with PTSD through analysis of interviews with primary care (PC) and mental health $(\mathrm{MH})$ clinicians. Our primary aims were to identify facilitators and barriers to PTSD guideline adherence regarding benzodiazepine prescribing to aid in the development of supportive strategies to de-implement this practice and improve the care of patients at increased risk of harms.

\subsection{Participants and setting}


To recruit participants, we selected VA Medical Centers based on hospital-level benzodiazepine data for patients with PTSD in fiscal years 1999- 2009 based on our larger work. ${ }^{5}$ We grouped all VA medical centers based on the degree of change in benzodiazepine prescribing rates in context to their initial frequency of prescribing into categories. Within each category 3 facilities were selected, considering geographic variance and number of veterans with PTSD treated. We had previously shown that rural patients treated at VA facilities had an increased likelihood of receiving benzodiazepines, ${ }^{16}$ so we selected at least one hospital in each group that was considered rural. We also noted geographic variation in prescribing benzodiazepines, so we selected hospitals to represent specific geographic regions. In total 12 VA Medical Centers and 2 smaller rural Community Based Outpatient Clinics were selected for inclusion in the study. Providers from 1 hospital did not respond to recruitment emails, resulting in 13 hospitals in the final sample. We asked $\mathrm{MH}$ leadership from selected hospitals to recommend the names of prescribing clinicians who treat PTSD in their general mental health $(\mathrm{MH})$ and primary care $(\mathrm{PC})$ clinics. We then randomly selected clinicians (1 from $\mathrm{MH}$ and $1 \mathrm{PC}$ ) from each hospital to participate. In total, we contacted 42 participants by email to recruit for participation, 8 failed to respond and 8 declined given time constraints, resulting in a total of 26 clinicians for a $68 \%$ participation rate.

\subsection{Ethical considerations}

After description of the study, all participants gave their informed consent. The Dartmouth College Committee for the Protection of Human Subjects and the White River Junction Vermont VA Research and Development Committee approved the procedures for this study (CPHS\# 22745).

\subsection{Data Collection}

Two clinical psychologists (EB and NB), trained in qualitative interviewing, conducted the telephone interviews throughout 2012. The psychologists alternated roles, with one serving as the lead interviewer and the other serving as back-up, taking notes and ensuring all interview topics were covered. A research assistant observed all interviews. The initial interview asked close-ended questions such as the clinician's department and length of VA employment. We then began the second part of the interview by asking prescribing clinicians to describe their overall management plan for a veteran with PTSD. (See Table 1 for topics of the Interview Guide most pertinent to this study.) Other interview topics included knowledge of the CPG and access to evidence-based PTSD psychotherapies. Professional transcriptionists produced verbatim transcripts and the research staff performed quality checks prior to analysis.

\subsection{Data Analysis}

The researchers reviewed verbatim transcripts and identified broad themes drawing on the interview guide topics to serve as top-level codes. Examples of top-level codes included factors influencing provider's rationale for current treatments as well as those factors influencing treatments that were avoided. Using inductive and deductive analysis with constant comparison methods that Parker and colleagues developed for similar qualitative studies, 3 members of the interview team (EB, NB, AJ) each coded one-third of the transcripts and reviewed $100 \%$ of each other's work. ${ }^{16}$ We created sub-codes by reviewing the quotes associated with each top-level code. Each interviewer took one-third of the quotes and applied the sub-codes. A secondary coder reviewed $25 \%$ of the sub-codes and discrepancies were discussed and resolved. We used Atlas.ti V6.2 qualitative data management software to assist with coding and analysis.

Results from the closed-ended questions were included for descriptive purposes only.

\section{RESULTS}

Demographic information for the participants is presented in Table 2. Several key themes emerged regarding factors that contributed to either new prescriptions of benzodiazepines or maintenance of existing prescriptions in veterans with PTSD. We categorized prominent barriers and facilitators as organizational, provider, and patient factors (See Table 3). Beliefs around these factors were similar between PC and MH providers. Where they are not, we describe these differences. Identified facilitators contributed to both decreased incidence and prevalence of benzodiazepine prescriptions, suggesting that such factors could help de-implement 
the use of benzodiazepines by reducing new prescriptions as well as renewal prescriptions.

\subsection{Barriers to Guideline-Concordant Benzodiazepine Use in Veterans with PTSD}

3.1.1 . Organizational barriers. The most common organizational barrier mentioned by both primary care and mental health prescribing clinicians was "inheriting" large caseloads of patients on chronic benzodiazepines. Many clinicians placed responsibility of initial prescriptions onto "older" prescribing clinicians who retired. Clinicians discussed facing caseloads that included many older-era veterans with PTSD maintained on benzodiazepines and unwilling to give them up. For example, a psychiatrist based in Mental Health reported, "I could almost see a patient and just look at what meds they are on and make a good guess as to which older physician had been treating them."

Although most prescribing clinicians who had been in their jobs for a decade on average agreed that the inherited caseloads of patients on benzodiazepines were problematic, they did not agree on how to treat them. Some clinicians who reported that they never or rarely write initial benzodiazepine prescriptions, argued they did not necessarily believe that discontinuation or tapering for the older "inherited" patients was imperative. They questioned the necessity of trying to taper the patient if it appeared that the patient was doing well for the time-being.

We've got a few prescribers [who have] been around here a while. It seems like everybody they see is on something like that or they're continuing or starting it. A lot of it is the older prescribers and the patient already has 100\% disability. They get started on these medications and at this point, it's not going to hurt them to stay on them. [Psychiatrist, $\mathrm{MH}$ ]

Some clinicians reported that they did not feel their facility leadership supported efforts to decrease the prescribing of benzodiazepines.

In the past, if you had a patient who was unhappy with you, they would just run upstairs to the 'QUAD' and talk to the leadership secretary. Next thing you know, I'm getting call saying either give them the benzo or give them another provider. [Physician's Assistant, $\mathrm{MH}$ ]

Most primary care providers said they believe that it is chiefly mental health's responsibility to make the decision to maintain or taper benzodiazepines and questioned the necessity of discontinuation in older veterans who are doing well. This is in part because primary care providers believe they do not have the time or resources to devote to this time-intensive work.

I don't want to step on Mental Health's area and yet there is so much of an overlap between what we do and what they do. But in these areas, like maybe sleep problems, where it is kind of theirs and kind of mine, there's sort of a song and dance there. I send a lot of emails suggesting maybe you consider prescribing something else. [Physician, PC]

Organizational factors such as documentation burdens and decreased time prescribing clinicians now have available with their patients may also play a role.

We need more time with the patients and the VA seems to be continually adding new screens and forms and making it harder for us. It's sort of like you are running a 400-meter dash and they want you to run faster, but they then keep adding 5-kilogram weights to the backpack you are carrying. [Psychiatrist, $\mathrm{MH}$ ]

3.1.2. Provider barriers. Provider factors that contributed to both increased incidence and prevalence of benzodiazepine prescriptions included the belief that there are complex symptoms best managed by these agents. Sleep and anxiety problems were prominent symptoms mentioned in the interviews as reasons to prescribe benzodiazepines but none of the providers intended to keep patients on them for long-term. There were concerns that depression and anxiety associated with PTSD increase the risk of suicide and that a benzodiazepine would decrease that risk.

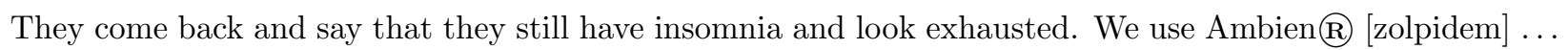
and that will be for sleep; then trazodone is our backup medication. For anxiety, then I'll use lorazepam and 
explain to the patient that it is temporary, we don't plan to keep them on it for years. [Psychiatrist, MH] I do sometimes prescribe clonazepam for someone who has suicidal thoughts. There is evidence that using something like clonazepam or an atypical can take the edge off the anxiety and help with suicide. So, I don't use it a lot, but I do when that is a concern. [Psychiatrist, $\mathrm{MH}$ ]

Prescribing clinicians mentioned that they occasionally use benzodiazepines to "kick-start" an SSRI, particularly in Primary Care where patients cannot be seen again for several months. Providers said that they use the benzodiazepines until they can get the patient into a psychotherapy treatment. Providers also report that they have their own concerns about the guideline-recommended antidepressants. They indicated concerns about patients not receiving full therapeutic dosage levels and about how these drugs might affect women, especially in pregnancy.

One of the things that we sometimes struggle to know is whether they have had an adequate trial before they've been moved on to other medications, or other medications have been added on top of the SSRI." [Psychiatrist, MH] Pharmaceutical and medical fields have not given strong and consistent recommendations about these agents, in particular SSRIs, in pregnancy, using them, not using them, which to use, which not to use, etc. So it is really nervous-making." [Psychiatrist, MH]

Finally, clinicians mentioned a lack of awareness about other effective treatment options in their own hospitals. They discussed discomfort about a severe "type" of PTSD, lack of knowledge regarding complex comorbidities, and the fact that the CPG does not go far enough to help. The providers made specific requests for education and guidance in these areas. This need was especially true for those new to the VA healthcare system and those within $\mathrm{PC}$ rather than $\mathrm{MH}$.

Initially, I was just looking for some guidance. I was rather confused just first coming to the VA and trying to understand where you start treating somebody with PTSD pharmacologically. [Psychiatrist, MH] There's also the factor that no one on my team I really feel is competent to do that [follow-up with patient by phone regarding medication management] as well as far as I could ask a nurse to do that for diabetes. I don't even go there with my nurse trying to figure out what to do with the PTSD. [Physician, PC]

3.1.3 . Patient barriers. Patient factors that the clinicians identified included patient requests for benzodiazepines and concerns about antidepressants side effects. First and foremost was the concern that for many patients, antidepressant medications are stigmatizing. Younger patients did not want to be on long-term medication.

I think one of the biggest obstacles as far as - well, not necessarily initiating SSRIs, other than the patient being hesitant to start a med if they have never been on one before- I think there's a lot of stigma associated with the antidepressant medication to this day. [Behavioral Health Pharmacist, PC]

Finally, because the US VA system provides benefits for service-connected disabilities there may be perceived economic disincentives for deprescribing medications. Many veterans fear that if they show improvement indicated by a reduction in medications, they will lose their benefits.

Who knows how true it is, but truth doesn't matter; it's the rumor that exists, that creates this scare of somebody even documenting just in the notes... of somebody getting better and this person having their benefits reduced. [Psychiatrist, $\mathrm{MH}$ ].

Although this may appear to be a VA specific problem, it could generalize to any system that serves patients whose healthcare coverage depends on a disability or other type of diagnosis such as American patients who receive Social Security Disability payments and associated medical insurance. Of course, in countries where there is universal healthcare, this barrier would not arise.

\subsection{Facilitators of Guideline-Concordant Benzodiazepine Use in Veterans with PTSD}

3.2.1. Organizational facilitators. Organizational factors that contributed to decreased prescribing of benzodiazepines for PTSD include consistent facility treatment practices and research presentations such as in journal clubs that present up to date findings. 
Fortunately, the other psychiatrists in this clinic, we're all on the same page regarding that, so there is consistency throughout the clinics which makes it easier when we have substance-seeking. [Psychiatrist, $\mathrm{MH}$ ]

Some clinicians reported that flexibility with their schedules to have time to use shared decision making practices and discuss safer options is critical; they rarely write initial benzodiazepine prescriptions. Another organizational factor that helped decrease benzodiazepine prescribing was integrated MH clinical teams that include psychologists and geriatric specialists who facilitated safer treatments for veterans.

I feel very lucky to work as closely as I do with the psychologists here and to have an integrated team so that when these treatment questions come up, the patient can talk about it with the psychologist and me, discuss cognitive behavioral therapy, and formulate a treatment plan. [Psychiatrist, $\mathrm{MH}$ ]

3.2.2 . Provider facilitators. Clinicians discussed strategies they use including psychoeducation with the patient to discuss potential harms and working to build rapport. For example, a prescribing clinician in Primary Care noted "A lot of this involves educating the veteran about medications and how they work. With benzodiazepines I discuss the risks of how they can be habit-forming and things like that."

The funny thing about the VA is, while your patient can fire you, you really can't fire your patient. And so... meaning, you just create another problem for somebody else if that person walks out of that meeting with you... So, I guess I just keep trying to build that trust. [Psychiatrist, MH]

Prescribing clinicians in both PC and MH were aware of the CPG recommendation against chronic benzodiazepine use in patients with PTSD and felt supported by it.

I think that it is helpful that the PTSD Clinical Practice Guideline is so clear about benzodiazepines and it's nice to be able to provide that with certain providers that have had a difficult time understanding that. [Psychiatrist, $\mathrm{MH}$ ] Obviously being a prescriber, I will talk to them about the medication options and be very frank about the limitations of medications, in the sense that they can help with some depression and anxiety, but at least in the context of starting an SSRI as a first-line agent. But I'm always planting the seed of how helpful psychotherapy can be, and then we can get more into that discussion, so I'm always pushing that. [Psychiatrist, MH]

3.2.3. Patient facilitators. The most often cited patient facilitator was established rapport and trust in the prescriber.

... you need to spend time with them to keep them engaged because on the first visit if the impression they have is not good, they're not coming back, they go back to alcohol. [Psychiatrist, MH, Medical Director for PTSD].

Patients' reports of unwanted symptoms can provide an entrée for providers to being discussions about reducing dosages.

It depends on what they complain about as far as symptoms that allows me to get that hook - if they're complaining of attention, concentration and memory, which they usually do, that is usually sometimes an appeal to try a lower dose. [Psychiatrist, MH].

\section{DISCUSSION}

This national qualitative study of VA prescribing clinician examined perspectives regarding the use of chronic benzodiazepines in veterans with PTSD. It is encouraging that compared to work done with community physicians, ${ }^{13}$ VA clinicians acknowledge problems associated with benzodiazepine overuse. Judging from the VA's decreased utilization rate, progress has been made. ${ }^{4}$ However we still see veterans with PTSD at increased risk of the harmful effects chronically prescribed these medications. ${ }^{4}$ In this study, we identified factors that contribute to new prescriptions as well as maintenance among both $\mathrm{MH}$ and PC clinicians that can help us understand how not just VA but community healthcare systems might design future efforts to promote change. 
Unfortunately, many clinicians and patients have found benzodiazepines effective, are unaware of harmful side effects and have not attributed adverse impacts to them. Caseloads of patients on benzodiazepines get passed on to new providers when a prescribing clinician leaves, and the new provider doesn't have time to taper these patients. Specific protocols that address how to handle inherited caseloads and education about complicated PTSD with multiple comorbidities will help providers make safer, informed decisions. Enlisting the help of clinical pharmacists to support patient tapers through these protocols can ease the burden on busy clinicians. Some providers, however, are still not convinced that benzodiazepines pose risks and, in some cases, believe there are continued benefits, particularly among older veterans who appear stable. Outreach education to these providers, to facility leadership and directly to the veterans and families to share recent research findings can improve this knowledge gap. Finally, several providers mentioned prescribing benzodiazepines to decrease suicidality. Suicide is indeed a significant risk for this population especially among older veterans; the majority of suicides in 2016 in VA occurred in those over $55 .{ }^{17}$ Evidence suggests, however, that rather than reduce suicide, there is an almost 3-fold increase in suicide risk in PTSD patients prescribed benzodiazepines. ${ }^{18}$ Including this information in educational materials for prescribing clinicians and sharing through direct mail with patients and families is critical.

Fortunately, the practice guideline recommends treatments that offer several safer effective alternatives. One solution to the benzodiazepine problem is to increase the use of SSRIs and/or trauma-focused psychotherapies that help target anxiety and insomnia while reducing overall PTSD symptoms. SSRIs are the recommended first-line treatment for anxiety and there are effective cognitive-behavioral therapy (CBT) protocols. ${ }^{19}$ The efficacy of brief CBT for insomnia, CBT-I , continues to grow and favors its use over many pharmacotherapies. ${ }^{20}$ Clinicians in this study reported they often do not offer psychotherapy as an option because they are unaware of its availability in their facility and because of administrative difficulties in access and consult processes. ${ }^{21}$ If providers are to benefit from effective psychotherapy alternatives, then administrative solutions such as a consult direct to a CBT-I provider could ease access problems. It is also critical that specialty care clinicians communicate with prescribing clinicians about the treatments available and encourage referrals.

We heard from clinicians that consistent care across services regarding the use of benzodiazepines made it easier for them to turn down requests for renewals or new prescriptions. Collaboration between prescribing providers can foster a consistent guideline concordant standard of care. Currently, although $\mathrm{MH}$ specialists who best understand the risks of benzodiazepines, prescribe the majority (70-80\%) of these drugs ${ }^{22}$ there has been an alarming increase in benzodiazepine prescriptions by PC providers who may be less knowledgeable of the CPG. ${ }^{12}$ Coordinated care can help to avoid problems such as a patient successfully tapering only to see a different provider in another clinic or in the community to start the medication again.

Clinicians identified other organizational changes that can foster guideline concordant prescribing. For example, providers can ensure the sharing of psychoeducational strategies and brochures including direct-topatient educational materials that highlight potential harms,${ }^{23,24,25}$ methods to engage patients and build trust, tapering clinics and success stories of patients who have lowered their dose, switched to a safer, longacting agent, or tapered from benzodiazepines. ${ }^{26}$ Instituting journal clubs and online courses that share new knowledge about PTSD psychopharmacology including SSRIs can help change the prescribing culture in a facility as well as sharing team approaches to assist with deprescribing. Finally, reducing benzodiazepines in veterans with PTSD aligns with national VA suicide prevention efforts. It is therefore critical that clinicians make appropriate clinical risk-benefit decisions concerning prescribing of these medications.

\section{Limitations}

There are several limitations to our findings that we should note. First, our intent was to describe barriers and facilitators participants face in the use of benzodiazepines for PTSD, not to judge the correctness of individual decisions. Some use of benzodiazepines may be justified but the trend of continued prescribing in what might be considered "high risk" subgroups such as older patients warrants additional actions. Second, patients' perspectives are critical. We had to rely on provider feedback about patient level barriers and facilitators, so patient voices should be reflected in future research. Finally, our findings are most applicable 
to providers working with military veterans who suffer from PTSD and are treated in the US VA health care system. As we observed above, however, we believe there are lessons learned from this work to help inform overall clinical decision making, especially for large national health systems which share some structural features with VA.

\section{Conclusion}

Due to the growing risks associated with benzodiazepines and their lack of efficacy in the treatment of PTSD, it is worthwhile for the VA and other healthcare systems to implement strategies that address the barriers and facilitators to guideline concordant treatment identified through this work. To do this, we must find ways, whenever possible, both to encourage tapering among patients already on these drugs and limit new prescriptions. Effective strategies to taper benzodiazepines exist but are time-consuming and providers need support to implement them. ${ }^{27}$ Hospital leadership needs to support culture changes and dissemination of evidence-based practices to encourage taking on this difficult task. VA has undertaken several steps to improve PTSD treatment practices that include dissemination of evidence-based psychotherapy, consultation for complex comorbid cases through the PTSD Consultation Program, and funding PTSD pharmacotherapy research to improve prescribing practices and discover new, safer alternatives to benzodiazepines. It will be essential as we continue to move forward to monitor the progress of these steps and the impact on care, particularly in older patients at greatest risk and who are least likely to be offered first-line psychotherapy treatments for PTSD. ${ }^{17}$ Ultimately, the results of these efforts should result in continued decreases in utilization of these medications and improved access to effective treatment alternatives among all patients with PTSD.

\section{DECLARATION OF CONFLICTING INTERESTS}

The author(s) declared no conflicts of interest concerning the research, authorship, and/or publication of this article.

\section{ACKNOWLEDGEMENTS}

The authors would like to thank the prescribing clinicians in VA hospitals who took part in this research. This project was supported by the Mental Health QUERI, Department of Veterans Affairs (MH-QUERI) (RRP 11001). Dr. Lund received additional support from the VA Health Services Research and Development Service (CDA 10-017; REA 09-220). None of these sponsors had any role in the study design, methods, analyses, and interpretation, or in preparation of the manuscript and the decision to submit it for publication. The views expressed in this article are those of the authors and do not necessarily reflect the position or policy of the Department of Veterans Affairs.

\section{REFERENCES}

1. Wells D, Popish S, Kay C, Torrise V, Christopher M. VA Academic Detailing Service: Implementation and Lessons Learned. Fed Pract. 2016; May 33(5):38-42.

2. Montano M, Bernardy NC, Sherrieb K. Cultivating change door to door: Educational outreach to improve prescribing practices in rural veterans with posttraumatic stress disorder. Sub Abuse, 2017;38:129134.

3. Program focuses on safe psychiatric medication. Department of Veterans Affairs (online). Available at www.blogs.va.gov/Vantage/27099/program-focuses-safe-psychiatric-medication. Accessed May 10, 2020.

4. Psychotropic Drug Safety Initiative Dashboard Report: Internal VHA data from the Corporate Data Warehouse, analysis by the VA Office of Mental Health and Suicide Prevention. Accessed June 13, 2019.

5. Lund BC, Bernardy NC, Alexander B, Friedman M. Declining benzodiazepine use in veterans with posttraumatic stress disorder. J Clin Psychiatry 2012;73:292-296.

6. U.S. Department of Veterans Affairs, \& U.S. Department of Defense. VA/DoD clinical practice guideline for the management of posttraumatic stress disorder and acute stress disorder.2017. Available at: 
https://www.healthquality.va.gov/guidelines/MH/ptsd/VADoDPTSDCPGFinal012418.pdf. Accessed March 1, 2020.

7. Guina J, Rossetter SR, DeRhodes BJ, Nahhas RW, Welton RS. Benzodiazepines for PTSD: A systematic review and meta-analysis. J Psychiatr Pract 2015; 21:281-303.

8. National Center for Veterans Analysis \& Statistics. Veterans population projection model FY17. 2017; Veterans Health Administration.

9. Penninkilampi R, Eslick GD. A systematic review and meta-analysis of the risk of dementia associated with benzodiazepine use, after controlling for protopathic bias. CNS Drugs, 2018; 32:485-497.

10. Yaffe K, Vittinghoff E, Lindquist K, et al. Posttraumatic stress disorder and risk of dementia among US veterans. Arch Gen Psychiatry, 2010;67:608-613.

11. Obiora E, Hubbard R, Sanders RD, Myles PR. The impact of benzodiazepines on occurrence of pneumonia and mortality from pneumonia: A nested case-control and survival analysis in a population-based cohort. Thorax. 2013;68:163-170.

12. Agarwal SD, Landon BE. Patterns in outpatient benzodiazepine prescribing in the United States. JAMA Network Open, 2019;2:e187399.

13. Cook JM, Marshall R, Masci C, Coyne JC. Physicians' perspectives on prescribing benzodiazepines for older adults: A qualitative study. J Gen Intern Med 2007;22:303-307.

14. Jessell L, Stanhope V, Manuel J, Mateu-Gelabert P. Factors associated with benzodiazepine prescribing in community mental health settings. Jrl of Sub Abuse Treatment 2020;109:56-60.

15. Lund BC, Abrams TE, Bernardy NC, Alexander B, Friedman M. Benzodiazepine prescribing variation and clinical uncertainty in treating posttraumatic stress disorder. Psychiatr Serv, 2013:64(1):21-27.

16. Parker LE, Ritchie MJ, Kirchner JE, Owen RR. Balancing health care evidence and art to meet clinical needs: Policymakers' perspectives. J Eval Clin Pract 2009;15:970-975.

17. Karel MJ, Wray LO, Adler G, et al. Mental health needs of aging veterans: Recent evidence and clinical recommendations. Clin Ger 2020, online 23 Jan.

18. Deka R, Bryan CJ, LaFleur J, Oderda G, Atherton A, Stevens V. Benzodiazepines, healthcare utilization, and suicidal behavior in veterans with posttraumatic stress disorder. J Clin Psychiatry 2018;79(6):17m12038.

19. National Institute for Health and Clinical Excellence. Management of anxiety (panic disorder with or without agoraphobia, and generalized anxiety disorder) in adults in primary, secondary and community care. London: NICE guidelines 2004 (amended 2007). http://www.nice.org.uk/Guidance/CG22.

20. Qaseem A, Kansagara D, Forciea MA, et al. Management of chronic insomnia disorder in adults: A clinical practice guideline from the American College of Physicians. Ann Intern Med, 2016;165,2:125133.

21. Barnett ER, Bernardy NC, Jenkyn AB, et al. Prescribing clinicians' perspectives on evidence-based psychotherapy for posttraumatic stress disorder. Behav Sci, 2014;4:410-422.

22. Abrams TE, Lund BC, Bernardy NC, et al. Aligning clinical practice to PTSD treatment guidelines: Medication prescribing by provider type. Psychiatr Serv, 2013;64:142-148.

23. Mendes MA, Smith JP, Marin JK, et al., Reducing benzodiazepine prescribing in older veterans: A direct-to-consumer educational brochure. Fed Pract, 2018;35:36-43.

24. Bernardy NC, Montano M, Cuccurrullo LA, Breen K, Cole, B. Taking the message to the rural patient: Evidence-based PTSD care. Jrl of Beh Med, In press.

25. Tannenbaum C, Martin P, Tamblyn R, et al. Reduction of inappropriate benzodiazepine prescriptions among older adults through direct patient education: The EMPOWER cluster randomized trial. JAMA Intern Med., 2014;174(6):890-898.

26. Benzodiazepines and PTSD [VA PTSD website], Available at https://www.ptsd.va.gov/understand_tx/benzos_ptsd.asp. Accessed August 15, 2020.

27. Morin CM, Bastien C, Guay B, et al. Randomized clinical trial of supervised tapering and cognitive behavioral therapy to facilitate benzodiazepine discontinuation in older adults with chronic insomnia. Am J Psychiatry, 2004;161:332-342. 


\section{Hosted file}

Table1_ClinicalDec. Making.pdf available at https://authorea.com/users/380388/articles/496399clinical-decision-making-regarding-benzodiazepine-use-in-ptsd-treatment

\section{Hosted file}

Table2_ClinicalDecMaking.pdf available at https://authorea.com/users/380388/articles/496399clinical-decision-making-regarding-benzodiazepine-use-in-ptsd-treatment

\section{Hosted file}

Table3_ClinicalDecMaking.pdf available at https://authorea.com/users/380388/articles/496399clinical-decision-making-regarding-benzodiazepine-use-in-ptsd-treatment 